\title{
Factores psicosociales y síndrome de Burnout en académicos de una universidad pública de México
}

\section{Psychosocial Factors and Burnout Syndrome in Academics of a Public University from Mexico}

\author{
Daniela Villamar Sánchez \\ Universidad Autónoma del Estado de Morelos, Cuernavaca, México \\ ORCID: https://orcid.org/0000-0002-5843-9628 \\ Arturo Juárez García* \\ Universidad Autónoma del Estado de Morelos, Cuernavaca, México \\ ORCID: https://orcid.org/0000-0003-3264-679X \\ Irma Gpe. González Corzo \\ Universidad Autónoma del Estado de Morelos, Cuernavaca, México \\ ORCID: https://orcid.org/0000-0002-9904-3873 \\ Mabel Osnaya Moreno \\ Universidad Autónoma del Estado de Morelos, Cuernavaca, México \\ ORCID: https://orcid.org/0000-0002-5369-0506
}

Recibido 01-07-19 Revisado 15-07-19 Aprobado 10-09-19 En línea 12-09-19

*Correspondencia

Email: arturojuarezg@hotmail.com

\section{Citar como:}

Villamar, D., Juárez, A., González, I., \& Osnaya, M. (2019). Factores psicosociales y síndrome de Burnout en académicos de una universidad pública de México. Propósitos y Representaciones, 7(3), 111 - 140. Doi: http://dx.doi.org/10.20511/pyr2019.v7n3.360 


\section{Resumen}

El objetivo del estudio fue identificar la relación que existe entre los factores psicosociales del trabajo académico y el Síndrome de Burnout en una universidad pública de México En una muestra de 247 académicos de una universidad pública de México, se aplicó una batería en línea que estuvo conformada por una adaptación del cuestionario de Factores Psicosociales del Trabajo Académico (FPSIS), el Cuestionario para la Evaluación del Síndrome de Quemarse por el Trabajo para Profesionales de la Educación (CESQT-PE) y un apartado de sociodemográficos Las prevalencias altas y/o críticas de las dimensiones de Burnout rondaron el 30\% al mismo tiempo que más del 90\% mostraron altos niveles de ilusión en el trabajo. Los modelos de regresión lineal multivariada mostraron una relación estadísticamente significativa entre todos los factores psicosociales con la mayoría de las dimensiones del Burnout independientes al sexo, la edad o el estado civil, alcanzando varianzas explicadas entre el $16 \%$ y el $43 \%$. Los predictores más confiables fueron "problemas sociales y organizacionales" y "problemas con alumnos", los que también tuvieron mayor prevalencia $(40.9 \%$ y $34.8 \%$ respectivamente). Parecen coexistir dimensiones negativas del burnout y la Ilusión por el trabajo en alguna medida. Los factores psicosociales encontrados se vinculan claramente a las nuevas políticas de educación superior. Se recomienda implementar acciones a nivel institucional que contribuyan a mejorar las condiciones laborales y el bienestar, así como programas preventivos para disminuir el estrés laboral y mejorar la calidad de vida de los académicos.

Palabras Clave: Factores psicosociales; Burnout; Profesor de universidad; Universidad pública

\section{Summary}

The objective of the study was to identify the relationship between psychosocial factors of academic work and Burnout Syndrome in a public university in Mexico. In a sample of 247 academics from a public university in Mexico, an online battery was applied that was made up of an adaptation of the questionnaire Psychosocial Factors of Academic Work (FPSIS), the Questionnaire for the Evaluation of Burning Syndrome by Work for Educational Professionals (CESQT-PE) and a section on sociodemographics The high and/or critical prevalence of Burnout dimensions was around 30\% at the same time as more than $90 \%$ showed high levels of illusion at work. Multivariate linear regression models showed a statistically significant relationship between all psychosocial factors and most Burnout dimensions independent of sex, age or marital status, reaching explained variances between $16 \%$ and $43 \%$. The most reliable predictors were "social and organizational problems" and "problems with students", which also had higher prevalence (40.9\% and $34.8 \%$ respectively). Negative dimensions of burnout and Illusion for work seem to coexist to some extent. The psychosocial factors found are clearly linked to the new higher education policies. It is recommended to implement actions at the institutional level that contribute to improving working conditions and well-being, as well as preventive programs to reduce work stress and improve the quality of life of academics.

Kewwords: Psychosocial Factors; Burnout; University Professor; Public University

\section{Introducción}

En las últimas décadas, el mundo del trabajo ha experimentado una transformación importante en los diferentes contextos laborales, lo que ha significado el planteamiento de políticas que comprenden nuevas formas de organización del trabajo y división de las tareas que priorizan la maximización de la productividad en un nuevo mundo globalizado (Thornley, Jefferys y Appay, 2010). Las condiciones laborales actuales producto de ese contexto han representado para la mayoría de los trabajadores diferentes desafíos, condiciones laborales precarias y bajo presupuesto para enfrentarlas, lo que ha impactado la salud física, mental y emocional de los 
trabajadores, en particular, a través de padecimientos como el estrés laboral negativo (Leka, Griffiths y Cox, 2003).

Quiñones, Tapia y Díaz (2012) y Gallardo y Quintanar (2008), señalan que esos cambios en los entornos laborales han agregado nuevos factores psicosociales a los trabajos tales como: la intensificación de las demandas laborales, el uso de nuevas tecnologías y tipos de trabajo, la sobrecarga laboral, ambigüedad, subordinación múltiple (rendir cuentas a varios jefes, según los grupos de trabajo a los que pertenezca) y la evaluación continua centrada en la productividad. Como consecuencia de la presencia de estos aspectos en el contexto laboral, los trabajadores se ven afectados en diversas áreas de su vida: salud, bienestar, calidad de vida y ámbito extralaboral (Morales-Nápoles, 2011).

De acuerdo con la Organización Internacional del Trabajo (2010), los Factores Psicosociales del Trabajo (FPS-T) están presentes en los diversos tipos de trabajadores en todo el mundo y sus consecuencias pueden ser a nivel individual (salud y bienestar) y/u organizacional (absentismo y rendimiento de los trabajadores). En ese sentido, los Factores Psicosociales del Trabajo (FPS-T) han adquirido gran importancia y la OIT los define como:

[Las] interacciones entre el trabajo, su medio ambiente, la satisfacción en el trabajo y las condiciones de su organización, por una parte, y por la otra, las capacidades del trabajador, sus necesidades, su cultura y su situación personal fuera del trabajo, todo lo cual, a través de percepciones y experiencias, pueden influir en la salud y en el rendimiento y la satisfacción en el trabajo. (OIT, 1986, p.3).

$\mathrm{Al}$ respecto, Bakker et al. (2014) agregan que existen factores psicosociales generales, es decir factores que se pueden encontrar en casi cualquier trabajo (como trabajo bajo presión y autonomía), y otros que son específicos o propios del rol que desempeña el trabajador. Además, también señalan que aquellos factores psicosociales percibidos como negativos (demandas laborales) son los principales predictores (causas) del Burnout, principalmente cuando no se cuenta con recursos para hacer frente a esas demandas (Bakker et al., 2014)

En ese sentido, la universidad pública como campo laboral también se ha sometido a políticas globales, anteponiendo la productividad a la calidad en el trabajo y la salud de los profesores (Gallardo \& Quintanar, 2008; Walker, 2016). Cabe señalar que la evaluación de las tareas académicas surge de ese tipo de políticas y en la universidad se lleva a cabo a través de programas de evaluación y recompensa centrados en la productividad de los académicos, principalmente en investigación (Díaz, 1996; Vera y González-Ledesma, 2018). Como resultado, la universidad se ha convertido en un entorno de constantes cambios y exigencias laborales en aumento para los trabajadores académicos (Terán y Botero, 2011; Vera y González-Ledesma, 2018).

En ese sentido, algunos de los factores psicosociales negativos que se han identificado como propios de la profesión académica según Caramés-Balo (2001, citado en Pando-Moreno et al., 2006) son: carga física, sobrecarga mental, cantidad de tiempo durante el cual la persona debe elevar las respuestas en su memoria y factores ambientales.

Pando-Moreno et al. (2006) señalan que, de 7 factores psicosociales explorados en su investigación con trabajadores académicos, solo 3 se mostraron como factores psicosociales negativos (demandas laborales) y relacionados con el Burnout: a) carga de trabajo, b) contenido y características de la tarea, y c) papel del académico y desarrollo de la carrera.

Por su parte, Silva y Flores (2014) señalan que a partir de su estudio con académicos de una universidad pública de México, los factores psicosociales que destacaron en nivel alto fueron: Condiciones del lugar de trabajo (57.3\%), Interacción social y aspectos organizacionales (49\%), Características del trabajo (32.8\%) y Contenido del trabajo (31\%). 
Aguilar et al. (2011) y Sánchez y Martínez (2014) señalan que la sobrecarga de trabajo es un aspecto latente en los profesores-investigadores de la universidad pública en México, ya que incluye un sinfín de actividades extra a la docencia, entre las que destacan la investigación, la tutoría, la difusión del conocimiento y actividades administrativas, así otras tareas sustantivas a estas.

Martínez-López, Martínez y Méndez (2015) identificaron la sobrecarga laboral como un aspecto reflejado en su estudio en el que el $86 \%$ de los académicos que participó señaló trabajar en días de descanso y vacaciones. Además, el $60 \%$ manifestó trabajar más de 48 horas semanales (Martínez-López, Martínez y Méndez, 2015).

Asimismo, existen estudios que han reportado la presencia de demandas físicas latentes en este ámbito laboral. En ese sentido, Pando-Moreno, Castañeda-Torres, et al. (2006) encontraron que la verbalización constante (86.7\%) y el esfuerzo visual prolongado (71.1\%) eran los aspectos de mayor prevalencia en el trabajo académico y que, además, presentaban relación con el síndrome de Burnout. Martínez-López et al. (2015), a partir de su estudio realizado con 192 académicos, reportan que una de las demandas físicas más frecuente en este trabajo de acuerdo con el 58\% de los académicos de su muestra es permanecer sentado la mayor parte de la jornada laboral.

Conforme a lo anterior, se ha demostrado que los factores psicosociales negativos son potenciales desencadenantes de diversos procesos de deterioro en la salud como agotamiento, problemas psicosomáticos y distrés; así como de resultados negativos en la organización, por ejemplo, absentismo, intención de abandono y bajo desempeño laboral (Bakker et al., 2014). En ese sentido, Velázquez (2010) señala que el distrés crónico, el Burnout en particular, es uno de los problemas que más aqueja a los académicos en su entorno de trabajo y fuera de él, además de ser un mal que afecta la productividad de las organizaciones.

El Burnout es un indicador de estrés crónico, y el punto medio entre los estresores y sus consecuencias (Gil-Monte, 2003). De acuerdo con Gil-Monte (2003), la mayor incidencia del síndrome de Burnout se da en profesionales que desempeñan actividades asistenciales o sociales (como el caso de la academia), por lo que el deterioro de su calidad de vida laboral también conlleva repercusiones sobre la sociedad en general. Ortega-Loubon, Salas y Correa (2011) coinciden con lo anterior y señalan que el Burnout es un problema de salud pública que va en aumento y que no tan solo repercute en los trabajadores, sino también en la calidad del servicio que ofrecen.

El síndrome de Burnout se puede manifestar en falta de motivación, interés y responsabilidad en las personas respecto al desempeño de su trabajo (Carlin y Ruiz, 2010; Maslach, 1998; Schaufeli, Leiter, y Maslach, 2009). Además, el Burnout también puede desencadenar repercusiones físicas como enfermedades crónico-degenerativas en los trabajadores que lo padecen, entre las que destacan hipertensión, diabetes y trastornos psicológicos, así como infartos (Velázquez, 2010).

Las investigaciones en torno a la presencia del síndrome de Burnout se han realizado en diferentes profesiones, entre ellas se encuentran algunas pocas investigaciones con profesores universitarios, tanto en México como en otros países. Un estudio que da cuenta de ello es el realizado por Pando-Moreno, Aranda-Beltrán, Aldrete, Flores y Pozos (2006) en el Centro Universitario de Ciencias de la Salud (CUCS) de la Universidad de Guadalajara con una muestra de 144 profesores, en donde se encontró que la población estudiada mostró alto grado de prevalencia de Burnout con al menos una de las 3 dimensiones afectada (52.7\%). De ese porcentaje, el $37 \%$ presentó solo una dimensión afectada, el $11.6 \%$ presentó 2 dimensiones afectadas y el $4.1 \%$ de la población calificaron como afectados en las 3 dimensiones (PandoMoreno, Aranda-Beltrán, et al., 2006). 
En otro estudio realizado con 156 docentes universitarios de Zacatecas, México por Ruiz de Chávez, Pando-Moreno, Aranda y Almeida (2014) también encontraron una alta prevalencia de Burnout en esta población, donde el $63.5 \%$ de los profesores universitarios presentó al menos una de las 3 dimensiones afectada. De los cuales, el 35.3\% de los profesores presentó daño en una sola dimensión, el $22.4 \%$ en dos dimensiones y solo el $7.7 \%$ presentó las tres dimensiones afectadas.

Otra investigación reporta que en una universidad pública de México con una muestra de 234 académicos, solo el 21\% de ellos manifestó puntajes de Burnout (Magaña-Medina y SánchezEscobedo, 2013). Del mismo modo, Magaña y Sánchez-Escobedo (2008) estudiaron la presencia del síndrome de Burnout en profesores-investigadores de Yucatán, México y encontraron una prevalencia del $19 \%$ en nivel moderado.

Sin embargo, en otro estudio desarrollado también en México con una muestra de 185 profesores universitarios de una universidad privada de la ciudad de Guadalajara, México, se encontró que la prevalencia del Burnout, a partir del cuestionario MBI, fue del 38.9\% de la población estudiada con al menos una de las 3 dimensiones afectadas. Asimismo, en una reciente investigación realizada mediante el cuestionario MBI con toda la planta académica de profesores de tiempo completo de una universidad pública de Baja California, México, se reportan niveles medio-alto en las dimensiones de agotamiento y cinismo y bajo eficacia profesional, lo que significa afectación en las tres dimensiones y alta posibilidad de padecer Burnout (Brito, 2018).

Esto deja ver la disimilitud en prevalencias de Burnout entre estudios aun tratándose de la misma ocupación. En otros países se han encontrado tendencias semejantes, Por ejemplo, en un estudio realizado en Colombia con una muestra de 194 profesores universitarios, a partir del cuestionario $M B I$ se encontró que de manera general los profesores percibían un nivel medio de Burnout, así como de cada una de sus tres dimensiones (Viloria Marín y Paredes Santiago, 2002), mientras que en el estudio de Caballero et al. (2009) señalan que derivado de su estudio con 101 profesores universitarios de tiempo completo de la ciudad de Barranquilla, Colombia, encontraron una prevalencia del $9.9 \%$ de niveles altos de Burnout. Un aspecto relevante de este estudio es el hecho de que la mayoría de los académicos participantes (88.1\%) manifestaron un nivel alto de Ilusión por el trabajo. Este mismo resultado concuerda con lo reportado por el estudio de Velazquez (2018) con 80 académicos de una universidad pública de Morelos, quienes manifestaron altos niveles de Ilusión por el trabajo.

Hasta aquí es importante señalar que aunque los estudios del síndrome de Burnout son cada vez más frecuentes en los académicos, aún son pocos comparados con los estudios que comprenden otras poblaciones como las del área de la salud, en donde se han desarrollado la mayoría de los estudios referentes al síndrome de Burnout, al menos en México (Juárez-García, Idrovo, Camacho-Ávila y Placencia-Reyes, 2014).

Asimismo, se considera importante destacar que la escasa investigación respecto a los factores psicosociales específicos del trabajo académico y su papel como predictores de Síndrome de Burnout, no deja clara su relevancia, sobre todo cuando el instrumento más utilizado en estos estudios es el MBI (Maslach Burnout Inventory), a pesar de sus limitaciones psicométricas en población mexicana (Placencia, Camacho, Juárez, Ballinas y Hernández, 2015). Tampoco queda claro cuáles factores psicosociales tienen mayor impacto en el Burnout, pues las escalas utilizadas en esos estudios no son exclusivas o adaptadas a la ocupación específica de la academia y sus particulares demandas. Por otra parte, la evidencia existente no es contundente para demostrar el peso relativo de los factores de riesgo psicosocial en el Burnout en relación a los aspectos sociodemográficos como el sexo, la edad o el estado civil, pues es común que no se controle su efecto a pesar de su relación con el síndrome (Jiménez, Hernández y Gutiérrez, 2000; León-Rubio, León-Pérez y Cantero, 2013) 
Finalmente, conforme a lo anteriormente expuesto y con el fin de contribuir al estudio de los factores psicosociales y su vínculo con el síndrome de Burnout en los académicos, el objetivo de este estudio es identificar la relación que existe entre los factores psicosociales del trabajo académico y el Síndrome de Burnout una universidad pública de México.

\section{Método}

\section{Diseño}

El diseño de este estudio es no experimental de tipo transversal de alcance correlacional.

\section{Participantes}

El muestreo fue no probabilístico a conveniencia. A partir de una población de 16,881 profesores universitarios se seleccionaron únicamente a aquellos que se desempeñan como Profesores e Investigadores en los centros temáticos y regionales de la institución, por lo que se enviaron 1,825 invitaciones mediante correo electrónico y participaron 259 académicos, por lo que la tasa de respuesta fue del $14.19 \%$.

Los criterios de inclusión fueron: realizar actividades de investigación y docencia, ser un trabajador activo en la Universidad al momento del estudio, tener un contrato de tiempo completo, participar en algún programa estímulos (PRODEP, SNI, SEI, Programa de Estímulos al Desempeño del Personal Docente) debido a las exigencias que estos programas suponen para los académicos, aceptar participar en el estudio y firmar la carta de consentimiento informado. Los criterios de exclusión fueron: ser docentes en sabático, ser profesores con contrato distinto al tiempo completo, no participar en algún programa estímulo, no aceptar participar en el estudio y no firmar/aceptar la carta de consentimiento informado. El criterio de eliminación fue no contestar la batería de instrumentos o contestarla parcialmente. Finalmente, conforme a estos criterios se obtuvo una muestra de 247 académicos.

La muestra final estuvo distribuida de la siguiente manera: por 122 hombres (49. 4\%) y 125 mujeres $(50.6 \%)$, con una edad promedio de 49.28 años (Desviación estándar $=9.677$ ). Respecto al estado civil, 149 fueron casados (60.3\%), 53 solteros (21.5\%), 23 viven en unión libre (9.3\%), 18 están divorciados (7.3\%) y 4 son viudos (1.6\%). En nivel máximo de estudios se observa que 38 tienen maestría (15.4\%), 179 tienen doctorado (72.5\%), y 30 tienen postdoctorado (12.1\%). Respecto al centro universitario de adscripción 205 pertenecen a un Centro Temático $(82.9 \%)$ y 42 a un Centro Regional (17.1\%).

Conforme al nombramiento oficial, 240 participantes señalaron ser Profesor-Investigador (97.2\%) y 7 señalaron ser Técnico Académico (2.8\%). Sin embargo, todos se dedican a realizar actividades de investigación y docencia. El promedio de antigüedad en la institución fue de 19.32 años (Desviación estándar $=10.983$ ), aunque el promedio en el puesto actual fue de 8.81 años (Desviación estándar $=7.832$ ).

\section{Instrumento}

La batería estuvo conformada por dos instrumentos y un apartado de sociodemográficos que exploró el sexo, el estado civil y la edad de los participantes y otros respecto a la adscripción y nombramiento. A continuación, se describen los 2 instrumentos adicionales:

El cuestionario de Factores Psicosociales en el Trabajo Académico (FPSIS Académicos) de Silva (2006). Su versión original está compuesta por 7 dimensiones y 50 ítems valorados por una escala de 5 grados, tipo Likert, que estima la frecuencia con la que los participantes perciben los factores psicosociales en su trabajo $(0=$ Nunca, $4=$ Siempre $)$. Sin embargo, para el presente estudio se efectuó un análisis factorial exploratorio que garantizara la validez psicométrica de 
dicho instrumento, lo que se describe más a detalle en el procedimiento y resultados. Los resultados iniciales requirieron de ajustes en la escala original. Al final se obtuvo una versión adaptada que sirvió para los propósitos de la exploración de su asociación con el síndrome de Burnout. Dicha adaptación mantuvo 26 ítems con las 7 dimensiones que tuvieron que ser reclasificadas y renombradas como sigue: Problemas sociales y organizacionales, 7 ítems (ej. Existen problemas de comunicación sobre los cambios que afectan su trabajo), Jornada excesiva, 3 ítems (ej. Su jornada de trabajo se prolonga más de nuevo horas diarias), Espacios y materiales insuficientes, 4 ítems, (ej. El espacio donde trabaja es inadecuado para las labores que realiza), Problemas con alumnos, 3 ítems, (ej. Enfrenta problemas con el comportamiento de los estudiantes), Insatisfacción con la recompensa económica, 3 ítems, (ej. Está inconforme con el salario que recibe por el trabajo que realiza), Esfuerzo mental y físico, 4 ítems, (ej. Requiere alto grado de concentración), Riesgos bioquímicos, 2 ítems, (ej. Esta expuesto a polvos, gases, solventes o vapores) (Tabla 1 ).

Para su evaluación se sumaron los puntajes de cada apartado y se dividieron entre el número de ítems, adicionalmente se clasificaron en tres categorías: bajo, medio y alto de acuerdo con puntos de corte establecidos a partir del percentil 66 y 99. Los resultados de este análisis se pueden ver Tabla 2.

Para medir el Síndrome de Burnout se utilizó el Cuestionario para la Evaluación del Síndrome de Quemarse por el Trabajo en su versión para Profesionales de la Educación (CESQTPE). Este instrumento está conformado por 20 ítems distribuidos en 4 dimensiones (Gil-Monte, 2011): Ilusión por el trabajo: Deseo del individuo de alcanzar las metas laborales porque suponen una fuente de satisfacción personal. Desgaste psíquico: Agotamiento emocional y físico debido a que en el trabajo se tiene que tratar a diario con personas que presentan o causan problemas. Indolencia: Presencia de actitudes negativas de indiferencia y cinismo hacia los clientes de la organización (pacientes, alumnos, etc.). Culpa: Sentimientos de culpa que la persona tiene por el comportamiento y las actitudes negativas desarrolladas en el trabajo, en especial hacia las personas con las que se establecen relaciones laborales. El CESQT mide cada dimensión a partir de una escala de frecuencia de cinco grados que van de 0 (nunca) a 4 (Muy frecuentemente; todos los días) (Gil-Monte \& Noyola, 2011). Puntuaciones bajas de Ilusión por el trabajo y altas puntuaciones en Desgaste e Indolencia indican altos niveles de Burnout. La escala Culpa permite diferenciar entre dos perfiles "con culpa" y "sin culpa", pero no contribuye a la puntuación total del CESQT. En ese sentido, vale la pena aclarar que, para fines de este análisis únicamente se evalúan las 3 dimensiones que miden el Burnout. Cabe señalar que este instrumento ofrece la ventaja de superar las insuficiencias psicométricas de otros instrumentos de origen anglosajón (Gil-Monte, Unda y Sandoval, 2009).

Además, este instrumento cuenta con validez psicométrica del modelo de cuatro dimensiones en México, realizada con personal de la educación de nivel básico a partir del cual se concluye que el instrumento en su versión para profesionales de la educación (CESQT-PE), resultó un instrumento fiable y válido para evaluar el SQT en México. Sin embargo, este mismo instrumento ha sido utilizado por Velazquez (2018) en un estudio con académicos de una universidad pública del estado de Morelos, quien señala que todas las dimensiones del constructo manifestaron un valor de consistencia interna aceptable (>.7), pero que su uso debe ser cuidadoso ya que algunos ítems no aplican al contexto académico. Como medida precautoria y de manera similar al instrumento previamente descrito, también se realizó un análisis factorial exploratorio adhoc para este estudio, el cual confirmó la estructura teórica original del CESQT (no se muestra por razones de espacio), por lo que no hubo cambio alguno para su uso en análisis posteriores.

\section{Procedimiento}

Para la aplicación se estructuró una batería en línea a través de la plataforma SurveyMonkey®. Para su envió fue necesario obtener los correos electrónicos de los académicos de la Universidad de Guadalajara, los cuales se consiguieron en la página Web del PRODEP titulada "Cuerpos 
Académicos reconocidos por el PRODEP”. Esta lista es de acceso público y se puede ingresar a ella a partir de la siguiente liga: https://promep.sep.gob.mx/ca1/.

Como parte de las consideraciones éticas, se formuló una carta de consentimiento informado. A través de este documento se solicitó la participación voluntaria de los académicos, se garantizó la confidencialidad de su identidad y se les confirió la libertad de abandono de la encuesta en el momento deseado. Para ingresar a contestar el cuestionario fue necesaria la aceptación de la carta.

Para la recolección de datos se envió el cuestionario por correo electrónico a académicos de los diferentes centros universitarios de la institución en la que se llevó a cabo esta investigación. Los centros universitarios están clasificados de la siguiente manera: a) Temáticos: se refieren a aquellos espacios organizados y administrados por áreas del conocimiento y son seis (UdeG, 1994); y b) Regionales: son aquellos espacios mediante los cuales se brinda atención a las diferentes necesidades multidisciplinarias de la población de las diferentes regiones de Jalisco donde se encuentran situados y son nueve (UdeG, 1994).

La estrategia de análisis de resultados requirió de tres pasos. Como se mencionó anteriormente, en un primer paso se realizaron análisis factoriales exploratorios para confirmar la estructura dimensional de las encuestas utilizadas sin asumir su validez implícita. El análisis factorial exploratorio se realizó con el método de extracción de mínimos cuadrados no ponderados, rotación oblimin y se utilizó el criterio del análisis paralelo Horn junto con el criterio de interpretabilidad teórica para la definición de número de factores (Lloret et al, 2014). Se consideraron cargas factoriales mínimas a $\lambda=.40$. Asimismo, se calculó el alfa Cronbach $(\alpha)$ y omega de Mc Donald's $(\omega)$ como índices de confiabilidad, este último se consideró necesario debido a la heterogeneidad de las cargas factoriales (Dunn, Baguley y Brunsden, 2014), todo ello con el software JASP (JASP Team, 2018).

En un segundo paso se estimaron las prevalencias de los factores psicosociales y el síndrome de Burnout, y finalmente en el tercer paso, se llevaron a cabo análisis de regresión lineal múltiple por el método de entrada para cada componente del síndrome de Burnout como variable de resultado, incorporando como predictores a las variables sociodemográficas y los factores psicosociales del trabajo.

\section{Resultados}

Las exploraciones factoriales iniciales no permitieron confirmar la validez psicométrica y por tanto la viabilidad del uso de la Escala de Factores Psicosociales Del Trabajo Académico, sin embargo, se buscó una versión adaptada que permitiera cumplir los objetivos del presente estudio. Para ello se eliminaron ítems que no contribuyeron a la varianza o confiabilidad del instrumento, aquellos que mostraron complejidades factoriales o aquellos que simplemente rompían con una interpretabilidad teórica. Se llegó así a la versión de 26 ítems y 7 factores antes descrita, la que mantuvo coherencia teórica y niveles de confiabilidad aceptables en todas sus dimensiones, obteniendo rangos de .70 a .86 tanto en el coeficiente $\omega$ como en $\alpha$ (Tabla 1 ).

En lo que corresponde a la confiabilidad de las dimensiones del CESQT-PE, se encontró que Ilusión por el trabajo presentó $\alpha=.85$ y $\omega=.86$, para Indolencia el valor fue $\alpha=.75$ y $\omega=.74$, la dimensión Desgaste psíquico presentó $\alpha=.87$ y $\omega$ con el mismo valor y para Culpa $\alpha=.87$ y $\omega=.88$, lo que en general dejó ver una alta consistencia en este instrumento. 
Tabla 1.

Matriz factorial y Alfa de Cronbach de las dimensiones del cuestionario FPSIS adaptado.

\begin{tabular}{|c|c|c|c|c|c|c|c|c|}
\hline $\begin{array}{c}\text { ESCALA FACTORES PSICOSOCIALES } \\
\text { ACADÉMICOS } \\
\text { (numeración original de ítems) }\end{array}$ & 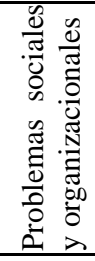 & 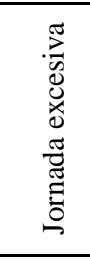 & 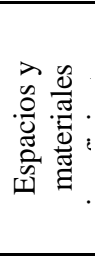 & 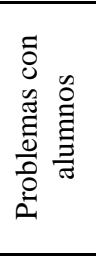 & 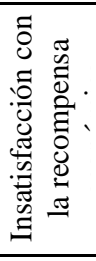 & 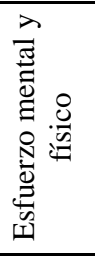 & 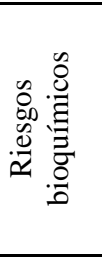 & 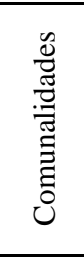 \\
\hline 16.10. Equipo y Materiales & .097 & .144 & .460 & .121 & .061 & -.091 & .142 & 0.535 \\
\hline 16.5. Polvos, Gases, etc. & .133 & .086 & -.067 & .037 & -.033 & .004 & .625 & 0.526 \\
\hline 16.6. Microbios, insectos, etc. & -.039 & -.061 & .063 & -.003 & .034 & -.000 & .879 & 0.212 \\
\hline 16.7. Carece de Cubículo & -.073 & -.115 & .574 & -.052 & .044 & -.085 & -.086 & 0.707 \\
\hline 16.8. Espacio Inadecuado & .008 & .031 & .746 & .013 & .035 & .007 & .017 & 0.396 \\
\hline 16.9. Hacinamiento & .086 & .017 & .703 & .006 & -.059 & .077 & .074 & 0.404 \\
\hline 17.1. Exceso de Actividades & -.042 & .600 & .098 & .055 & .051 & .174 & .047 & 0.485 \\
\hline 17.3. Jornada Prolongada & -.052 & .841 & .005 & .011 & .005 & -.056 & -.016 & 0.335 \\
\hline 17.4. Trabaja Fines y Vacaciones & .117 & .580 & -.025 & -.063 & .076 & .073 & -.065 & 0.581 \\
\hline 18.2. Problemas de Comportamiento Est. & -.039 & .052 & -.014 & .621 & .102 & -.015 & 0.043 & 0.582 \\
\hline 19.1. Tarea Compleja & .075 & -.025 & -.017 & .135 & -.041 & .617 & 0.010 & 0.572 \\
\hline 19.2. Grado de Concentración & -.011 & -.044 & .045 & -.023 & -.033 & 631 & -.013 & 0.624 \\
\hline 19.3. Posturas Incómodas & .017 & .264 & .111 & .007 & .108 & .484 & .071 & 0.495 \\
\hline 19.5. Esfuerzo Visual & .095 & .333 & -.098 & -.110 & .034 & .446 & .058 & 0.544 \\
\hline 20.2. No Valoran Educación & .086 & .031 & .008 & .751 & -.047 & .044 & -.000 & 0.382 \\
\hline 21.1. Relación con Compañeros & .421 & .024 & -.022 & .282 & -.133 & -.048 & .075 & 0.671 \\
\hline 21.3. Problemas con Jefe & .622 & .195 & .023 & .008 & -.165 & -.190 & .084 & 0.555 \\
\hline 21.4. Procesos de Evaluación & .437 & .012 & .106 & .170 & .252 & -.007 & -.057 & 0.537 \\
\hline 21.5. Sistemas de Control & .583 & -.021 & .034 & .058 & .135 & .106 & -.006 & 0.503 \\
\hline 21.6. Problemas de Comunicación & .773 & -.031 & .013 & -.022 & .091 & .060 & .068 & 0.288 \\
\hline 21.7. Limitación en Decisiones & .762 & -.021 & .082 & -.016 & -.011 & .136 & .036 & 0.293 \\
\hline 21.8. Información de Eficacia & .764 & -.043 & .006 & .033 & .069 & -.054 & -.046 & 0.413 \\
\hline 22.1. Inconforme con Salario & .027 & .035 & .093 & .008 & .719 & -.058 & -.058 & 0.437 \\
\hline 22.2. Modificación Sistema Pensiones & .004 & .067 & -.019 & .001 & .595 & -.014 & .210 & 0.539 \\
\hline 22.3. Programas de Estímulos & .160 & .005 & -.069 & .065 & .573 & .092 & .037 & 0.538 \\
\hline 24.3. Alumnos Insoportables & -.117 & -.100 & .032 & 637 & .032 & -.006 & -.012 & 0.611 \\
\hline \multirow[t]{2}{*}{ Confiabilidad } & .86 & .76 & .75 & .71 & .72 & .71 & .73 & \\
\hline & .86 & .75 & .74 & .70 & .72 & .70 & .73 & \\
\hline
\end{tabular}

Nota: Elaboración propia

Con respecto a la prevalencia, de manera general se puede decir que los factores psicosociales son percibidos en un nivel bajo, medio o alto de manera heterogénea por la mayoría de los académicos, sin embargo, es importante destacar aquellos que se perciben en un nivel alto. De manera más específica, los factores I) problemas sociales y organizacionales y IV) problemas con alumnos, fueron percibidos en un nivel alto por el $40.9 \%$ y $34.8 \%$ respectivamente, lo que los ubican con la más alta prevalencia en este estudio. No obstante, la suma de prevalencias en los niveles medio y altos deja ver que en todos los factores psicosociales existen riesgos medios o altos en más del $50 \%$ de los casos (Tabla 2). 
Tabla 2.

Prevalencias del nivel de percepción de los Factores psicosociales del trabajo académico

Factores psicosociales del trabajo académico

\begin{tabular}{|c|c|c|c|c|c|c|c|}
\hline Nivel & 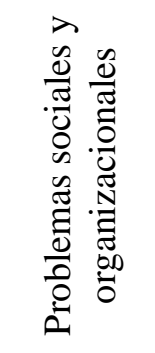 & 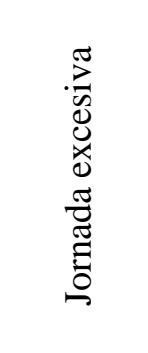 & 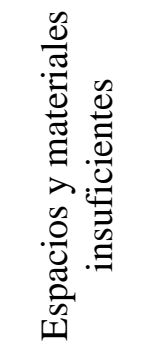 & 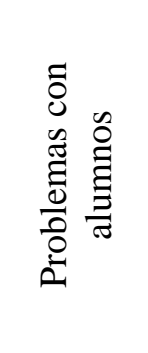 & 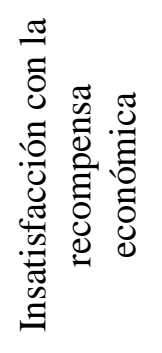 & 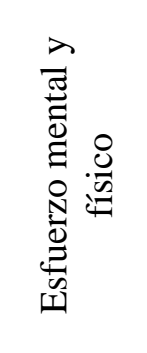 & 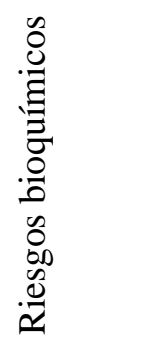 \\
\hline Bajo & $\begin{array}{c}72 \\
(29.1 \%)\end{array}$ & $\begin{array}{c}96 \\
(38.9 \%)\end{array}$ & $\begin{array}{c}104 \\
(42.1 \%)\end{array}$ & $\begin{array}{c}120 \\
(48.6 \%)\end{array}$ & $\begin{array}{c}81 \\
(32.8 \%)\end{array}$ & $\begin{array}{c}89 \\
(36 \%)\end{array}$ & $\begin{array}{c}87 \\
(35.2 \%)\end{array}$ \\
\hline Medio & $\begin{array}{c}74 \\
(30 \%)\end{array}$ & $\begin{array}{c}96 \\
(38.9 \%)\end{array}$ & $\begin{array}{c}63 \\
(25.5 \%)\end{array}$ & $\begin{array}{c}41 \\
(16.6 \%)\end{array}$ & $\begin{array}{c}90 \\
(36.4 \%)\end{array}$ & $\begin{array}{c}88 \\
(35.6 \%)\end{array}$ & $\begin{array}{c}78 \\
(31.6 \%)\end{array}$ \\
\hline Alto & $\begin{array}{c}101 \\
(40.9 \%)\end{array}$ & $\begin{array}{c}55 \\
(22.3 \%)\end{array}$ & $\begin{array}{c}80 \\
(32.4 \%)\end{array}$ & $\begin{array}{c}86 \\
(34.8 \%)\end{array}$ & $\begin{array}{c}76 \\
(30.8 \%)\end{array}$ & $\begin{array}{c}70 \\
(28.3 \%)\end{array}$ & $\begin{array}{c}82 \\
(33.2 \%)\end{array}$ \\
\hline
\end{tabular}

Nota: Elaboración propia.

Los resultados de la evaluación del CESQT-PE para conocer el nivel de percepción del Síndrome de Burnout por los académicos se presentan en la Tabla 3. Los resultados más relevantes señalan que respecto a la dimensión Ilusión por el trabajo, la mayoría de los académicos (96.8\%) la percibió en nivel "crítico", aunque debe considerarse que los puntajes para esta dimensión deben ser interpretados de manera invertida, bajas puntuaciones en esta dimensión indican altos niveles de Burnout, lo que significa que solo para este caso un nivel "crítico" es algo positivo, pues representa que la ilusión por el trabajo es muy alta.

En cuanto a la dimensión Indolencia, esta es percibida por la mayoría de los académicos (32\%) en un nivel medio, aunque también se observan porcentajes altos en los niveles bajo (21.5\%) y alto (22.3\%) para esta dimensión. La dimensión de Desgaste psíquico también es percibida en un nivel medio por la mayoría de los académicos (34.8\%), pero se obtuvieron porcentajes altos en los niveles bajo (21.1\%) y alto (20.6\%).

Finalmente, al evaluar la puntuación total de la escala CESQT-PE, se obtuvo que la mayoría de los académicos (33.6\%) percibieron el Burnout en un nivel medio, pero también se encontró un porcentaje alto $(30 \%)$ en el nivel bajo.

Tabla 3.

Frecuencias respecto al nivel de percepción del Síndrome de Burnout

\begin{tabular}{cccccc}
\hline Nivel & CESQT-PE & Ilusión & Indolencia & Desgaste & Culpa \\
\cline { 2 - 6 } Muy bajo & $7(2.8 \%)$ & $0(0 \%)$ & $42(17 \%)$ & $33(13.4 \%)$ & $62(25.1 \%)$ \\
Bajo & $74(30 \%)$ & $1(0.4 \%)$ & $53(21.5 \%)$ & $52(21.1 \%)$ & $41(16.6 \%)$ \\
Medio & $\mathbf{8 3 ( 3 3 . 6 \% )}$ & $3(1.2 \%)$ & $\mathbf{7 9 ( 3 2 \% )}$ & $\mathbf{8 6}(\mathbf{3 4 . 8 \%})$ & $\mathbf{7 4 ( 3 0 \% )}$ \\
Alto & $49(19.8 \%)$ & $4(1.6 \%)$ & $55(22.3 \%)$ & $51(20.6 \%)$ & $52(21.1 \%)$ \\
Crítico & $34(13.8 \%)$ & $\mathbf{2 3 9}$ & $18(7.3 \%)$ & $25(10.1 \%)$ & $18(7.3 \%)$ \\
& & $\mathbf{( 9 6 . 8 \% )}$ & & & \\
Total & $247(100 \%)$ & $247(100 \%)$ & $247(100 \%)$ & $247(100 \%)$ & $247(100 \%)$ \\
\hline
\end{tabular}

Nota: Elaboración propia

Con respecto a la relación entre el Síndrome de Burnout, los factores sociodemográficos (edad, sexo, estado civil) y los factores psicosociales del trabajo académico, los análisis de la regresión lineal múltiple mostraron algunas tendencias interesantes. En principio vale decir que 
todos los modelos fueron estadísticamente significativos, lo que dejó ver la contribución conjunta y relevante de las variables sociodemográficas y los factores psicosociales académicos a las variables del síndrome de Burnout, alcanzando varianzas explicadas en un rango de $16 \%$ al $43 \%$ (la R cuadrada ajustada -R2- más alta fue para indolencia).

En particular, resaltaron los factores de "problemas sociales y organizacionales" y "problemas con alumnos" como los predictores más consistentes en la mayoría de las variables del Burnout, con pesos propios e independientes a los aspectos sociodemográficos. En seguida en importancia, fue la jornada exhaustiva y el esfuerzo mental y físico, la primera se presentó como el principal predictor psicosocial o de mayor peso para el desgaste psíquico $(\beta=.273)$. Un caso inesperado es la relación de esfuerzo mental y físico con la ilusión en el trabajo, la que se muestra positiva y en dirección contraria a lo esperado teóricamente (a mayor esfuerzo, mayor ilusión), aunque esta relación fue baja $(\beta=.140)$ (Tabla 4$)$.

En cuanto a los aspectos sociodemográficos, la edad fue un predictor confiable para indolencia $(\beta=-.147)$ y parcialmente para culpa $(\beta=-.115)$ (mayor edad, menores puntajes de las variables de Burnout) y sexo lo fue para desgaste $(\beta=-.278)$ (los hombres tuvieron menos desgaste psíquico). En la Tabla 4 se resaltan las variables constituyentes del modelo que se asocian con las dimensiones del Síndrome de Burnout, el valor del R2 (normal y ajustado), el peso predictivo (b) y el Beta Tipificado $(\beta)$ de cada variable con su respectivo valor de significancia.

Tabla 4.

Análisis de regresión lineal de las dimensiones del Síndrome de Burnout y los Factores sociodemográficos y psicosociales del trabajo académico

\begin{tabular}{|c|c|c|c|c|c|c|c|c|c|}
\hline & $\mathbf{F}$ & $\mathbf{R 2}$ & $\begin{array}{c}\mathbf{R 2} \\
\mathbf{a}\end{array}$ & $b$ & $\begin{array}{r}\text { Error } \\
\text { estándar }\end{array}$ & $\beta$ & $p$ & $\begin{array}{l}\text { Límite } \\
\text { inferior }\end{array}$ & $\begin{array}{l}\text { Límite } \\
\text { superior }\end{array}$ \\
\hline Ilusión por el trabajo & 5.72 & .19 & .16 & & & & .000 & & \\
\hline \multicolumn{10}{|l|}{ Sociodemográficos } \\
\hline Sexo (masculino) & & & & -.045 & .083 & -.033 & .589 & -.209 & .119 \\
\hline Edad & & & & .008 & .004 & .110 & .069 & -.001 & .016 \\
\hline Edo. Civil (con par & & & & .166 & .090 & .111 & .066 & -.011 & .343 \\
\hline \multicolumn{10}{|c|}{ Factores psicosociales académicos } \\
\hline Problemas sociales & & & & -.266 & .061 & -.323 & .000 & -.387 & -.146 \\
\hline Jornada excesiva & & & & .030 & .051 & .039 & .555 & -.071 & .132 \\
\hline Espacios y materia & uficier & & & .002 & .044 & .003 & .961 & -.085 & .089 \\
\hline Problemas con alt & & & & -.153 & .064 & -.155 & .017 & -.279 & -.027 \\
\hline Insatisfacción de re & ensa e & nica & & .018 & .045 & .028 & .680 & -.069 & .106 \\
\hline Esfuerzo mental & & & & .124 & .060 & .140 & .039 & .006 & .242 \\
\hline $\begin{array}{c}\text { Riesgos } \\
\text { bioquímicos }\end{array}$ & & & & -.048 & .042 & -.077 & .249 & -.131 & .034 \\
\hline Indolencia & 19.58 & .45 & .43 & & & & .000 & & \\
\hline \multicolumn{10}{|l|}{ Sociodemográficos } \\
\hline Sexo (masculino) & & & & -.038 & .055 & -.034 & .494 & -.146 & .071 \\
\hline Edad & & & & -.008 & .003 & -.147 & .003 & -.014 & -.003 \\
\hline Edo. Civil (con par & & & & .015 & .059 & .012 & .804 & -.102 & .132 \\
\hline \multicolumn{10}{|c|}{ Factores psicosociales académicos } \\
\hline Problemas sociales & & & & .058 & .041 & .087 & .155 & -.022 & .138 \\
\hline
\end{tabular}




\begin{tabular}{|c|c|c|c|c|c|c|c|}
\hline Jornada excesiva & & -.035 & .034 & -.056 & .308 & -.102 & .032 \\
\hline Espacios y materiales insuficientes & & -.056 & .029 & -.106 & .056 & -.114 & .002 \\
\hline Problemas con alumnos & & .499 & .042 & .629 & .000 & .415 & .582 \\
\hline Insatisfacción de recompensa económica & & -.033 & .029 & -.063 & .261 & -.091 & .025 \\
\hline Esfuerzo mental y físico & & -.044 & .040 & -.063 & .264 & -.123 & .034 \\
\hline $\begin{array}{c}\text { Riesgos } \\
\text { bioquímicos }\end{array}$ & & .021 & .028 & .042 & .448 & -.033 & .076 \\
\hline $\begin{array}{lll}\text { Desgaste Psíquico } & 16.10 & .40\end{array}$ & .38 & & & & .000 & & \\
\hline \multicolumn{8}{|l|}{ Sociodemográficos } \\
\hline Sexo (masculino) & & -.553 & .104 & -.278 & .000 & -.757 & -.349 \\
\hline Edad & & -.008 & .005 & -.078 & .133 & -.019 & .002 \\
\hline Edo. Civil (con pareja) & & -.062 & .112 & -.029 & .580 & -.282 & .158 \\
\hline \multicolumn{8}{|l|}{ Factores psicosociales académicos } \\
\hline Problemas sociales y org. & & .181 & .076 & .152 & .019 & .031 & .331 \\
\hline Jornada excesiva & & .307 & .064 & .273 & .000 & .181 & .433 \\
\hline Espacios y materiales insuficientes & & -.030 & .055 & -.032 & .583 & -.139 & .078 \\
\hline Problemas con alumnos & & .250 & .079 & .175 & .002 & .094 & .407 \\
\hline Insatisfacción de recompensa económica & & .064 & .055 & .068 & .248 & -.045 & .173 \\
\hline Esfuerzo mental y físico & & .186 & .075 & .145 & .013 & .039 & .333 \\
\hline $\begin{array}{l}\text { Riesgos } \\
\text { bioquímicos }\end{array}$ & & .026 & .052 & .028 & .622 & -.077 & .128 \\
\hline $\begin{array}{lll}\text { Culpa } & 6.98 & .22\end{array}$ & .19 & & & & .000 & & \\
\hline \multicolumn{8}{|l|}{ Sociodemográficos } \\
\hline Sexo (masculino) & & .007 & .073 & .005 & .927 & -.137 & .150 \\
\hline Edad & & -.007 & .004 & -.115 & .052 & -.015 & .000 \\
\hline Edo. Civil (con pareja) & & -.052 & .079 & -.039 & .505 & -.207 & .102 \\
\hline \multicolumn{8}{|l|}{ Factores psicosociales académicos } \\
\hline Problemas sociales y org. & & .131 & .054 & .178 & .015 & .026 & .237 \\
\hline Jornada excesiva & & .134 & .045 & .192 & .003 & .045 & .222 \\
\hline Espacios y materiales insuficientes & & -.073 & .039 & -.123 & .062 & -.149 & .004 \\
\hline Problemas con alumnos & & .319 & .056 & .362 & .000 & .209 & .429 \\
\hline Insatisfacción de recompensa económica & & -.084 & .039 & -.143 & .032 & -.161 & -.007 \\
\hline Esfuerzo mental y físico & & -.094 & .052 & -.119 & .075 & -.197 & .010 \\
\hline $\begin{array}{c}\text { Riesgos } \\
\text { bioquímicos }\end{array}$ & & .024 & .037 & .044 & .504 & -.048 & .097 \\
\hline
\end{tabular}

\section{Discusión}

El objetivo de este estudio fue analizar la relación del Síndrome de Burnout con los factores psicosociales del trabajo académico considerando factores sociodemográficos. Primero, debe señalarse que la prevalencia del Síndrome de Burnout, respecto al nivel alto del 19.8\% (49) y crítico del $13.8 \%$ (34), resultó en $33.61 \%$ (83). Estos resultados tienen relativa correspondencia a los de Pando-Moreno et al. (2006) mediante el uso del Maslach Burnout Inventory (MBI) en dónde encontraron una prevalencia del $38.9 \%$ en una muestra de 185 profesores universitarios de una universidad privada de la ciudad de Guadalajara, México. En otro estudio llevado a cabo en una universidad pública de México, con una muestra de 234 académicos se reportó que el 21\% 
de los académicos manifestó puntajes sugerentes de Burnout (Magaña-Medina y SánchezEscobedo, 2013).

Además, un aspecto relevante es el hecho de que la mayoría de los académicos participantes (96.8\%) de este estudio manifestaron un nivel alto de Ilusión por el trabajo. Estos resultados concuerdan con los hallazgos de Caballero et al. (2009) en profesores universitarios en Colombia. Del mismo modo, Velazquez (2018) señala en su estudio con académicos de una universidad pública de Morelos, quienes manifestaron altos niveles de Ilusión por el trabajo.

Respecto a los factores psicosociales se encontró que los de mayor prevalencia en nivel alto fueron I) Problemas sociales y organizacionales y IV) Problemas con alumnos por el $40.9 \%$ y $34.8 \%$ de los académicos, respectivamente. Silva y Flores (2014) coinciden con lo anterior y señalan que Interacción social y aspectos organizacionales fue el segundo factor con mayor prevalencia (49\%) factor identificado en nivel alto por los académicos de su estudio. A diferencia del estudio de Pando-Moreno, Castañeda-Torres, et al. (2006) en donde reportan que los factores de mayor prevalencia en nivel alto fueron: Exigencias laborales y Remuneración al rendimiento en nivel alto con un $22.3 \%$ y $12 \%$, respectivamente. Estas diferencias pueden deberse al tipo de población estudiada, ya que este último estudio referido se realizó con profesores de una universidad privada, aunque también pudo ser resultado de la adaptación de la escala propuesta. La adaptación de la escala utilizada en esta investigación exploró y agrupó factores más específicos de la ocupación, como "problemas con alumnos" o "esfuerzos mentales y físicos", dejando atrás conceptos generales como "interacción social" o "exigencias laborales" utilizados en la escala original. Futuros estudios deberán valorar el aporte de esta versión más breve de la escala utilizada.

Respecto a la jornada laboral excesiva, Magaña-Medina, Aguilar-Morales, y SánchezEscobedo (2014) señalan que la sobrecarga laboral es un factor predominante en los profesoresinvestigadores de la universidad pública en México, debido a las diversas actividades que desempeñan adicional a la docencia como, investigación, tutoría, difusión del conocimiento y actividades administrativas, entre otras. Asimismo, Martínez-López et al. (2015) señalan que respecto a la jornada laboral el $86 \%$ de los académicos de su estudio señaló trabajar días de descanso y vacaciones, y el $60 \%$ señaló que trabaja más de 48 horas a la semana.

Es importante recalcar que las relaciones encontradas entre las dimensiones del Síndrome de Burnout con los factores psicosociales académicos evaluados: Problemas sociales y de organización, Problemas con alumnos, Esfuerzo físico y mental, Jornada excesiva e Insatisfacción con la recompensa económica, fueron independientes a los aspectos sociodemográficos, lo que es un aporte importante de la presente investigación, ya que son pocos los estudios de alcance multivariado en la literatura del Burnout en México (Juárez et al., 2014). En algunos estudios, aunque univariados, se han encontrado resultados similares respecto a la relevancia de los factores identificados en esta investigación. En relación al factor Jornada excesiva de este estudio y su relación con la dimensión de desgaste psíquico en población académica, Pando-Moreno, Castañeda-Torres, et al. (2006) encontraron que la carga de trabajo resultó ser un factor de riesgo para agotamiento emocional, aunque también encontraron relacionados los factores "verbalización constante" y el "esfuerzo visual prologado". Del mismo modo, Martínez-López et al. (2015), a partir de su estudio, identificó que el permanecer sentado la mayor parte de la jornada laboral es uno de los factores más prevalentes señalado por el 58\% de los académicos.

Un aspecto inesperado fue la relación positiva de esfuerzo mental y físico e ilusión en el trabajo, lo que pudiera deberse al esfuerzo de académicos que se comprometen demasiado con su trabajo de una manera positiva. Sin embargo, al mismo tiempo ocurre una correlación positiva del esfuerzo mental y físico con desgaste psíquico, lo que deja dudas respecto a la posible influencia de otras variables no controladas. En todo caso, el peso de esta variable fue muy bajo para considerarse importante. 
En concordancia con lo esperado, los factores Espacios y material insuficiente y Riesgos bioquímicos no tuvieron trascendencia en sus relaciones con el Burnout, al tratarse de una ocupación con poca exposición a esos aspectos. Estos resultados coinciden con lo reportado por Pando-Moreno, Castañeda-Torres, et al. (2006) respecto a la dimensión que ellos señalan como condiciones del lugar de trabajo y que es similar a los dos factores que aquí se señalan. La dimensión condiciones del lugar de trabajo no manifestó asociaciones estadísticamente significativas con ninguna dimensión del Burnout en su estudio.

Con todo lo anterior, se puede concluir que pese a que alrededor del $30 \%$ de los académicos presentan niveles altos o críticos en las dimensiones del Síndrome de Burnout, la mayoría presentan niveles considerablemente altos de Ilusión por el trabajo, lo que significa que tienen altos deseos de alcanzar las metas laborales y una fuente de placer personal por su trabajo (GilMonte, Carretero, Roldán y Núñez, 2005) al mismo tiempo que pueden coexistir momentos de desgaste, un tema que necesita mayor investigación.

La prevalencia de factores psicosociales más alta encontrada fue específicamente para problemas de comunicación y de organización, así como problemas con los alumnos, los que también fueron de mayor riesgo de acuerdo a su relación con el burnout, dichos factores se vinculan claramente a las nuevas políticas educativas nacionales e internacionales en el sentido que implican presiones diversas y problemas de comunicación generadas por procesos y sistemas de organización ligados a la productividad académica y el cumplimiento de programas de acreditación individual e institucional que cada vez son más agobiantes para los académicos e investigadores, que también están vinculados a el incremento de matrícula y el aumento considerable de estudiantes, con lo que aumenta el riesgo de demandas emocionales por la mayor interacción con estos.

Las asociaciones encontradas entre los diferentes factores psicosociales y el Síndrome de Burnout indican que todos los factores psicosociales tienen algún impacto al menos parcial en el Síndrome de Burnout, sobre todo con la dimensión de Desgaste psíquico, por ello la más idónea estrategia de prevención implica implementar acciones de mejoramiento psicosocial a nivel de la institución, a manera de que contribuyan a mejorar las condiciones laborales en general, ya que cinco de los siete factores psicosociales explorados se manifestaron en un nivel medio, y de acuerdo con la teoría se clasificarían como factores de "posible" riesgo psicosocial, y uno en nivel alto, el cual se clasificaría como factor "de riesgo" psicosocial.

Es importante que las nuevas políticas de educación superior incluyan el desarrollo de programas preventivos de bienestar que orienten a los académicos respecto al manejo del estrés laboral y de salud en general, con el propósito de prevenir y promover la calidad de vida académica, así como de la calidad de la educación superior y de las mismas instituciones, ya que son los académicos los principales actores encargados de la educación y la calidad en la misma.

\section{Referencias}

Aguilar, M. N., Magaña-Medina, D. E., \& Surdez, P. E. G. (2011). Satisfacción laboral en profesores investigadores universitarios. [Working Paper]. Recuperado de http://www.repositoriodigital.ipn.mx/handle/123456789/3823

Bakker, A., Demerouti, E., \& Sanz-Vergel, A. I. (2014). Burnout and Work Engagement: The JD-R Approach. Annual Review of Organizational Psychology and Organizational Behavior, 1, 389-411. doi https://doi.org/10.1146/annurev-orgpsych-031413-091235

Brito, L. J. (2018). Calidad educativa en las instituciones de educación superior : evaluación del síndrome de burnout en los profesores. Revista Iberoamericana Para La Investigación y El Desarrollo Educativo, 8(16), 516-534. doi: https://doi.org/10.23913/ride.v8i16.356

Caballero, D. C., González, G. O., Mercado, A. D., Llanos, B. C., Bermejo, B. Y., \& Vergel, M. C. (2009). Prevalencia del Síndrome de Burnout y su correlación con factores psicosociales en docentes de una institución universitaria privada de la ciudad de Barranquilla. Psico, $12(21), 142-157$. 
Carlin, M., \& Garcés de los Fayos, R. E. (2010). El síndrome de burnout: Evolución historica desde el contexto laboral al ámbito deportivo. Anales de Psicología, 26(1), 169-180.

Díaz, B. A. (1996). Los programas de evaluación (estímulos al rendimiento académico) en la comunidad de investigadores. Un estudio en la UNAM. Revista Mexicana de Investigación Educativa, 1(2), 408-423. Recuperado de http://www.redalyc.org/articulo.oa?id=14000209

Gallardo, A., \& Quintanar, K. (2008). La flexibilidad del trabajo académico. Problemática en la universidad pública. Revista Administración y Organización, 9(17), 55-65.

Gil-Monte, P. R. (2003). El síndrome de quemarse por el trabajo (síndrome de Burnout) en profesionales de enfermeria. Revista Eletrônica InterAção Psy, 1(1), 19-33. Recuperado de http://www.bvsde.ops-oms.org/bvsacd/cd49/artigo3.pdf

Gil-Monte, P. R. (2011). Evaluación del cuestionario CESQT. Madrid.

Gil-Monte, P. R., Carretero, N., Roldán, M. D., \& Núñez, R. E. M. (2005). Prevalencia del síndrome de quemarse por el trabajo (burnout) en monitores de taller para personas con discapacidad. Psicología Del Trabajo y de Las Organizaciones, 21(1-2), 107-123. Recuperado de http://www.redalyc.org/articulo.oa?id=231317039007

Gil-Monte, P. R., \& Noyola, C. V. (2011). Estructura factorial del "Cuestionario para la Evaluación del Síndrome de Quemarse por el Trabajo" en maestros mexicanos de educación primaria. Revista Mexicana de Psicología, 28(1), 75-84. Recuperado de https://www.researchgate.net/publication/262675381\%250AEstructura

Gil-Monte, P. R., Unda, S. R., \& Sandoval, O. J. I. (2009). Validez factorial del « Cuestionario para la Evaluación del Síndrome de Quemarse por el Trabajo » ( CESQT ) en una muestra de maestros mexicanos. Salud Mental, 32(3), 205-214. Recuperado de http://www.scielo.org.mx/scielo.php?script=sci_arttext\&pid=S0185-33252009000300004

Jiménez, B. M., Hernández, E. G., \& Gutiérrez, J. L. G. (2000). La evaluación del estrés y el burnout del profesorado: el CBP-R. Revista de Psicología Del Trabajo y de Las Organizaciones, 16(2), 151-171.

Juárez-García, A., Idrovo, Á., Camacho-Ávila, A., \& Placencia-Reyes, O. (2014). Síndrome de burnout en población mexicana: Una revisión sistemática. Salud Mental, 37(2), 159-176. doi: https://doi.org/10.17711/SM.0185-3325.2014.020

Leka, S., Griffiths, A., \& Cox, T. (2003). Work organisation and stress: systematic problem approaches for employers, managers and trade union representatives. Recuperado de https://www.who.int/occupational_health/publications/en/oehstress.pdf

León-Rubio, J. M., León-Pérez, J. M., \& Cantero, F. J. (2013). Prevalencia y factores predictivos del burnout en docentes de la enseñanza pública: el papel del género. Ansiedad y Estrés: Una Revista Multidisciplinar de Psicología, Medicina, Neurociencias y Ciencias Sociales, $1,11-25$.

Magaña-Medina, D. E., Aguilar-Morales, N., \& Sánchez-Escobedo, P. (2014). Burnout among research teams: Evidence from mexican "Cuerpos Academicos." Business Education and Acreditation, 6(2), 35-46.

Magaña-Medina, D. E., \& Sánchez-Escobedo, P. (2008). Síndrome de desgaste emocional en Investigadores Mexicanos. International Journal of Psychology, 42(2), 353-362. Recuperado de http://www.redalyc.org/articulo.oa?id=28442216\%250ACómo

Magaña-Medina, D. E., \& Sánchez-Escobedo, P. (2013). Burnout and Role Ambiguity in Mexican Universities: A case study. Revista Educación y Ciencia, 3(41). Recuperado de https://www.researchgate.net/publication/307205342_Burnout_and_Role_Ambiguity_in_ Mexican_Universities_A_case_study

Martínez-López, C. R., Martínez, A. S., \& Méndez, R. I. (2015). Estímulos económicos, exigencias de trabajo y salud en académicos universitarios mexicanos. Salud de Los Trabajadores, 23(1), 5-18. Recuperado de http://www.scielo.org.ve/scielo.php?script=sci_arttext\&pid=S1315-01382015000100002

Maslach, C. (1998). Burnout. Enciclopedia de Salud y Seguridad En El Trabajo, p. 3.74-3.75.

Morales-Nápoles, E. (2011). Factores psicosociales en el trabajo: Reflexiones conceptuales, teoricas y metodologicas. In A. Juárez-García \& A. Camacho-Ávila (Eds.), Reflexiones teorico-conceptuales de lo psicosocial en el trabajo (Primera, pp. 163-186). Cuernavaca, Mor.: Ediciones Mínimas. 
Organización Internacional del Trabajo (OIT). (1986). Factores psicosociales en el trabajo: Naturaleza, incidencia y prevención (Primera). Ginebra: Organización Internacional del Trabajo.

Organización Internacional del Trabajo (OIT). (2010). Riesgos emergentes y nuevos modelos de prevención en un mundo de trabajo en transformación.

Ortega-Loubon, C., Salas, R., \& Correa, R. (2011). Aspectos epidemiológicos del síndrome de burnout en el personal sanitario. Hospital Aquilino Tejeira. Archivos de Medicina, 7(24). Recuperado de http://www.redalyc.org/pdf/503/50319889001.pdf

Pando-Moreno, M., Aranda-Beltrán, C., Aldrete, R. M. G., Flores, S. E., \& Pozos, R. E. (2006). Factores psicosociales y burnout en docentes del centro universitario de ciencias de la salud. Investigación En Salud, 8(3), 173-177. Recuperado de http://www.medigraphic.com/pdfs/invsal/isg-2006/isg063f.pdf

Pando-Moreno, M., Castañeda-Torres, J., Gregoris-Gómez, M., Aguila-Marín, A., Ocampo-deAguila, L., \& Navarrete, R. M. (2006). Factores psicosociales y síndrome de burnout en docentes de la Universidad del Valle de Atemajac, Guadalajara, México. Salud En Tabasco, 12(3), 523-559. Recuperado de http://www.redalyc.org/articulo.oa?id=48712304

Quiñones, M., Tapia, T., \& Díaz, C. (2012). El Rol de das Demandas y los Recursos Laborales en la Salud Mental de Trabajadores Chilenos del Sector de Servicios. 14(45), 201-2010.

Ruiz de Chávez, D., Pando-Moreno, M., Aranda, B. C. B. C., \& Almeida, P. C. (2014). Burnout y Work Engagement en Docentes Universitarios de Zacatecas. Ciencia \& Trabajo, 16(50), 116-120. doi: https://doi.org/10.4067/S0718-24492014000200010

Sánchez, L. C., \& Martínez, A. S. (2014). Condiciones de trabajo de docentes universitarios, satisfacción, exigencias laborales y daños a la salud. Salud de Los Trabajadores, 22(1), 1926. Recuperado de http://www.scielo.org.ve/scielo.php?script=sci_arttext\&pid=S131501382014000100003

Schaufeli, W., Leiter, M., \& Maslach, C. (2009). Burnout: 35 years of research and practice. Career Development International, 14(3), 204-220. doi: https://doi.org/10.1108/13620430910966406

Silva, G. B. N. (2006). Factores piscosociales y desagaste profesional en académicos del Centro Universitario de Ciencias Biológicas y Agropecuarias. Universidad de Guadajara.

Silva, G. B. N., \& Flores, R. V. (2014). Académicos universitarios y salud ocupacional . Importancia de los factores psicosociales y variables sociodemográficas, el caso de un centro universitario regional de la Universidad de Guadalajara, México. Revista Población y Desarrollo: Argonautas y Caminantes, 10.

Terán, R., \& Botero, Á. (2011). El capitalismo organizacional: una mirada a la calidad de vida laboral en la docencia universitaria. Cuadernos de Administración, 27(46), 9-21.

Thornley, C., Jefferys, S., \& Appay, B. (2010). Globalization and precarious forms of production and employment: Challenges for workers and unions (C. Thornley, S. Jefferys, \& B. Appay, Eds.). Edward Elgar Publishing.

Velazquez, B. N. (2018). El Burnout (SQT) como factor psicosocial de riesgo en profesores de la Universidad Autónoma del Estado de Morelos. Universidad Autónoma del Estado de Morelos.

Velázquez, V. D. (2010). Estrés, empleo y salario de los académicos de la educación superior. Matices, 5(13). Recuperado de http://www.revistas.unam.mx/index.php/matices/article/view/25792

Vera, H., \& González-Ledesma, M. A. (2018). Calidad y evaluación: matrimonio del cielo y el infierno. Perfiles Educativos, 40(Especial), 53-97. doi: https://doi.org/10.22201/iisue.24486167e.2018.Especial.59180

Viloria Marín, H., \& Paredes Santiago, M. (2002). Estudio Del Síndrome De Burnout O Desgaste Profesionalen Los Profesores De La Universidad De Los Andesa Study of Burnout Syndrome or Professional Fatigue in Professors in the University of Los Andes. Educere, 6(17). Recuperado de http://www.redalyc.org/html/356/35601704/

Walker, V. S. (2016). El trabajo docente en la universidad : condiciones, dimensiones y tensiones. Perfiles Educativos, 38(153), 105-119. Recuperado de http://www.scielo.org.mx/scielo.php?script=sci_arttext\&pid=S0185-26982016000300105 